\title{
Living with a Chemically Sensitive Wife: A "We" Situation
}

\author{
K. Hutton Carlsen, A. M. Topp, and S. Skovbjerg \\ Department of Dermato-Allergology, The Danish Research Centre for Chemical Sensitivities, Copenhagen University Hospital Gentofte, \\ 2900 Copenhagen, Denmark
}

Correspondence should be addressed to K. Hutton Carlsen, katrina-musen@hotmail.com

Received 17 August 2012; Accepted 27 September 2012

Academic Editors: C. Banwell, E. Clays, A. Slep, and R. Spiewak

Copyright ( $) 2012$ K. Hutton Carlsen et al. This is an open access article distributed under the Creative Commons Attribution License, which permits unrestricted use, distribution, and reproduction in any medium, provided the original work is properly cited.

\begin{abstract}
Objectives. Multiple chemical sensitivity (MCS) is a medically unexplained and socially disabling disorder characterized by negative health effects attributed to exposure to common airborne chemicals. How spouses of chemically sensitive women experience and cope with their partners illness has not been described previously. Methods. This study presents data from three semistructured focus group interviews with a selective sample of 13 men whose spouses had MCS. Data was analyzed using systematic text condensation with a special focus on coping. Results. The informants expressed a great concern for their wives and described their role in the relationship as that of a "watchdog", always alert and ready to protect. A considerable amount of time and effort was thus given to avoid symptom-eliciting chemicals to prevent illness. Informing guests of restrictions and observing neighbours' daily routines were common occurrences. Masks, installation of additional air filters in cars and houses, and other protective measures had necessitated several participants taking on extra work to alleviate the economic burden. The wives' illness had thus become a "we" situation. Discussion. In conclusion, MCS was perceived as a "we" situation and may thus impose considerable strain on the relationship and the family as a whole.
\end{abstract}

\section{Introduction}

Multiple chemical sensitivity (MCS) is a medically unexplained and socially disabling disorder characterized by negative health effects attributed to exposure to common airborne chemicals, for example, fragranced products, tobacco smoke, freshly printed papers or magazines, or new furniture [1]. Symptoms are generally attributed to previous chemical exposures and recur on a subsequent exposure to the same or structurally unrelated chemicals at levels normally considered to be nontoxic [1]. The reported symptoms typically vary between individuals with women being more sensitive and reporting more symptoms than men do [25]. A typical symptom pattern is thus difficult to establish. However, nonspecific central nervous system (CNS) complaints are frequently reported including fatigue, headache, and difficulty in concentrating [2, 3]. Other symptoms include pain and respiratory complaints [2,3]. More theories have been proposed to explain MCS, biological as well as psychological $[1,6]$, but the pathophysiology is still poorly understood. MCS is one of many terms used to describe this disorder and will, without reference to any assumptions about aetiology, be used here.

The number of population-based studies on symptoms attributed to common airborne chemicals is limited and has yielded different prevalence estimates according to the case definitions applied in the studies. Prevalence estimates in these studies range from $9-33 \%[2-5,7,8]$. In contrast, prevalence estimates of physician-diagnosed MCS or reports of disabling consequences in the form of social and occupational disruptions range from $0.5-6.3 \%[2,3,5,8]$. Knowledge of the risk factors involved in the development from mere annoyance to a debilitating state of illness is sparse. However, results from a recent population-based prospective study suggest that increased levels of stress and strain may be risk factors in the development of chemical sensitivity [9].

The unpredictability of MCS and absence of observable evidence of pathology is a key difficulty which chemically sensitive persons and their families have to cope with. 
Avoidance is the coping strategy that is most often used by affected individuals [10]. However, avoidance may have a considerable impact on their daily lives and the lives of family, friends, and other social networks [11-13], ultimately resulting in restricted flexibility in personal life, less social contact, relational strain, and difficulties in maintaining a job or in finding a suitable workplace. To be able to socialize, chemically sensitive persons often depend on other peoples' willingness to adjust their lifestyles to meet their needs for a chemical-free environment. The consequences may be experiences of rejection and loss of social network [11, 13, 14]. The lifestyle changes associated with MCS are likely to also interfere with the spouses' lives, adding additional strain on the relationship. In a study on spouses' perspectives on living with a chronically ill person, more than half of the spouses reported that it had strained their personal life situation in one way or another [15]. In terms of finances there may be increased expenses for families affected by MCS, for example, new cars with extra and more efficient air filters, new housing, isolation from neighbours emitting smoke from open fires or wood burning stoves, masks worn to prevent exposures, extra clothes wash, and the purchase of fragrance-free products [16]. Job loss or reduction in working hours is likely to lead to an insecure financial situation [14], and spouses may become the main source of income and support. It has been described that living with a chronically ill person may lead to a change of roles in the family in terms of responsibility and workload [17]. However, rather than the illness itself, the coping strategies applied by both spouses and patients appear to play an important role in overall satisfaction with the marriage [18]. How spouses of chemically sensitive women experience and cope with the changes in daily life often associated with MCS has, to the best of our knowledge, not been described previously.

\section{Objectives}

The objectives of this study were to describe how spouses of chemically sensitive women experience and cope with their partners' illness and the lifestyle changes associated with this disorder.

\section{Materials and Methods}

Data for this study was drawn from three 90-minute focus group interviews at the Danish Research Centre for Chemical Sensitivities, Department of Dermato-allergology, Copenhagen University Hospital Gentofte. The interviews were conducted over a period of two months and were moderated by the principal author.

A selective sampling strategy was applied, recruiting spouses of women with at least one-year duration of MCS and who had children in order to secure a common framework. Information on the women was available through a research database at the Research Centre. Possible informants were invited to participate through letters of invitation. Altogether forty-five men received a letter of invitation and fifteen men subsequently agreed to participate in the focus groups; however, two declined shortly before the interviews because of sickness.

A semistructured interview guide was developed to guide the focus group discussions. The following themes were explored: the influence of MCS on the marital relationship and daily life in the family and the coping strategies applied within the family. The interviews were audio-taped and later transcribed verbatim by the principal author. The transcripts included informant interventions, stammering, faltering, and any break in articulation, to give a detailed picture of whether the subject was difficult. All authors read the transcribed interviews, and the analyses were conducted in collaboration. Data analysis was performed based on the principles described by Kvale and Halkier $[19,20]$ : first, all transcripts were read several times to obtain an overall impression of the material; then units portraying the participants' experiences with MCS in daily life were identified and categorized, guided by the aim of the study; the content of the identified categories was then condensed, followed by a crosscheck to validate the findings. All names stated in the section "Results" are fictive in order to secure anonymity.

\section{Approval}

The study was approved by the Danish Data Protection Agency. Under Danish legislation, focus groups do not require approval from an ethics committee.

\section{Results}

The informants were aged 37-69 years with an average age of 59 years. Four had children living at home, two had children in kindergarten, and two had teenagers. MCS had been an intricate part of the informants' lives for between 7 and 40 years.

Four main themes were identified: (1) the home, (2) economic consequences, (3) relationships with family and friends, and (4) relationships with neighbours and strangers. The four themes are presented in the following text.

5.1. The Home. Overall, the informants described a difficult life situation, where substantial changes and adaptations had been made to support and cope with their wives' illness. As one expressed: "When I say we, it is because it is something which affects me so much that it is a "we" situation, is not it? (Steven).

5.1.1. A "Watchdog". The informants expressed concern for their wives. To avert illness, many changes had been made at home and in daily routines. Preventive actions included showering and changing clothes when returning from work, reading newspapers outdoors to prevent the smell from freshly printed paper entering the house, installing additional air filters in the car to eliminate petrol fumes, and even moving to a new home to escape the smoke from neighbours' open fires or wood burning stoves. 
The informants described themselves as "watchdogs," always on guard to protect their wives from being exposed to symptom-eliciting agents that would make them ill.

..I've had the experience that I've been a bl... watchdog for many years, I have not exactly gone on in front, but I've been able to say that there is something here, put your mask on (William)

As one man expressed: "she" (his wife) should really have a watchdog with her to point her in the right direction" (Casper).

The informants sometimes had to act quickly to help their wives avoid an oncoming threat. Often, the decision was made not to go out at all, preferring to stay at home because it was easier.

5.1.2. Being Powerless. The informants expressed feelings of powerlessness and found it difficult to understand why everyday products had to contain fragrance. Wind direction was another important issue. Flags had been hung outside to register the direction of the wind to determine whether windows should be closed or opened to ventilate or cool the house. Large fans had been installed to ventilate the house if a troublesome odor should intrude. Reprimands and arguments were unavoidable if windows or doors had not been closed in time, especially during the summer when neighbours mowed lawns or lit barbecues. When something reminded the men of an earlier incident, they were prompted to be proactive and tried to avoid it by using earlier solutions, for example, asking neighbours to inform them when grass was to be mowed or barbecues lit.

One informant stated: "It's almost impossible to predict these things, like being one step ahead, you know, that's the worst, you'd like to protect your wife in some way but you feel powerless, you cannot catch everything and avert it. Sometimes you can with something but not... that's the frustration" (William).

New houses, cars, and furniture emit odors and gasses; accordingly, either they had to be degassed before being used or purchased second hand. New electronic devices also presented problems, resulting in purchasing second-hand TVs, computers, and so forth, or the alternative: nothing! In some cases, the informants had installed outdoor kitchens as their wives were bothered by strong food smells. Some did all the household chores alone while others did only the chores that made their wives ill, for example, cleaning, shopping, cooking, or emptying the dishwasher. One of the informants described a family dilemma: "We could not cope with my neighbour's oil-fired boiler. Because...sigh...I had to make an agreement with him. I had to buy him a new gas boiler" (William). The alternative was moving to a new neighbourhood to avert the problem.

5.1.3. The Children. The informants described how their children found it difficult to cope with the limitations they encountered. Computers were prohibited in some of the informants' homes because these devises could elicit symptoms of MCS. It was also difficult for the children to abstain from using fragranced products when all their friends used them. Likewise, on returning home, some had to change their clothes outside and bathe. Several men used a socialsupport strategy by utilising the children's assistance, for example, the youngsters helped buy clothes for their mothers and aired or washed the garments.

5.2. Economic Consequences. The changes in lifestyle and even housing, described in the previous section, were expensive for the families involved and led to financial concerns.

\section{... and then we have cars with extra filters...so we drive with masks and filters... we have a house in Sweden, bought solely because of MCS and people ask Why the (swear word..)... we want to live there, and I say, do you know what, one kilometre that way there is a neighbour and 3/4 kilometre in that direction there is a neighbour. She really relaxes there (Christoffer)}

As the majority of the wives received disability pensions, the men had to generate the major part of the household income. They were concerned about their financial situation and to make ends meet, some had taken extra jobs or had done so before retiring.

\section{My wife is a disabled pensioner ... so errr... it's me who has to earn money, right (Casper)}

The men's role in the family was accompanied by many limitations. One participant recounted that it was impossible for him to change jobs because it would be difficult for him to find another job with a similar income:

I can easily recognise that with (clears his throat), that is, I have also, my wife has also because of MCS gone into early retirement... then it is only my income apart from those crumbs, that is in fact, it's only now I realize that I'm tied to the job I have now (Steven)

\subsection{Mastering Relationships with Family and Friends}

5.3.1. Staying Home. Relationships with family and friends were paramount, and considerable effort was put into making them work. The informants described sending a telephone text message or e-mail reminding guests that fragranced products were not allowed in the house, and to avoid misunderstandings or conflicts, guests received a brochure with facts about MCS. If, despite these instructions, a guest had used a fragranced product, s/he was asked to shower and was offered an alternative set of clothes. "Yes, we have clothes in all sizes" (Christoffer) as one informant commented. Refusal to comply or forgetfulness resulted in some guests being asked to sit in another room, or in arranging an alternative day to visit.

5.3.2. Invited Out. When the wives were invited out, they tended to refuse, and when the wives refused, the husbands tended to do likewise. The husbands felt guilty about leaving 
their wives alone at home. If the husbands did decide to accept an invitation, they were usually away from home for only a short period as they were aware that their wives were already very isolated. People were usually invited into the MCS family's own safe surroundings. When the couple ventured out, it was to close family and friends only, and for only a short time.

5.3.3. Loss of Friends. In many cases, these precautions had the inevitable consequence that friendships were dropped. "It's better to part with people who fail to understand the consequences of their actions and their lack of understanding than to spend time and energy trying to make them grasp it" (Emil), as one man in the group explained, and the other informants agreed. Apart from family and friends, the men also had the responsibility of dealing with relationships with neighbours and strangers.

\subsection{Mastering Meetings with Neighbours and Strangers}

5.4.1. Neighbours. When addressing new neighbours, "strange" behavior (wearing of masks, constant airing of clothes, changing of clothes outside the house, etc.) was explained and the necessary educational information was conveyed. Invitations were also equipped with the same information, as a reminder. Some neighbours were understanding and considerate by agreeing to refrain from wearing fragranced products or by mowing lawns at predetermined times, thus allowing windows and doors to be closed in advance, while others displayed complete lack of understanding. Neighbours' comings and goings in their cars and the weekly refuse collection lorry were also a problem: "It is especially difficult when dealing with strangers who have no knowledge of the problem" (Michael).

5.4.2. Strangers. One remarked: "If we are in a public place or in the cinema or theatre and they see my wife with a mask on, they immediately think that she has a contagious disease and almost flee across the backs of the chairs" (Tom). The informants found it frustrating to see the reactions their wives provoked. Shops and supermarkets were also a problem. Not only because other shoppers wore fragranced products, but also because many shops used fragrant aromas in the ventilation system to create an atmosphere. These aromas cause considerable inconvenience.

Direct confrontation with the shop owners and an explanation had resulted in these aromas being removed, in some cases. Another informant recounted problems in an airport where it was impossible to avoid the odors from the fragrance department and would, together with another participant, contact the airport to find a solution.

While on holiday, one informant had informed people around them of his wife's problem, hoping to minimize their use of fragranced products; another informant had bought fragrance-free products for all their acquaintances and had asked them to use similar products in the future.

In places where it was not possible to be proactive, for example, cinemas and theatres, the informants always tried to purchase aisle seats to minimize the risk of chemical exposure. If aisle seats were not available, they would try to swap seats or would attend an early performance where the audience is generally smaller.

Overall, the informants felt powerless in public places as they constantly had to play the part of a watchdog.

\section{Discussion}

This focus group study is the first to describe how husbands of chemically sensitive women experienced and coped with the many changes to daily living often associated with MCS. Our findings showed that extensive changes in daily lives are made to cope with the situation, and spouses play a very active part, that is, it has become a "we" situation. Most striking is perhaps the reference to the "watchdog," which emphasizes the alertness and attentiveness these spouses display and which has become an integrated part of their lives. Although the informants in the focus groups gave the impression of commitment and strong relationships with their wives, it was obvious from the discussions that the changes in daily living were a strain on the family and had a large impact on their lives as well.

The strategies described by the informants relate to both problem-focused and emotion-focused coping. Purchasing second-hand computers to avoid evaporation from new electronic devises or reminding guests in advance that fragranced products were not allowed in the house are examples of problem-focused strategies. The informants experienced that these strategies in some cases resulted in loss of contact with friends and family. Proactive mastering was also employed, constantly attempting to be one step ahead of problems, that is, being the "watchdog." The frustration of not being able to control strangers in public places often resulted in using avoidance as a coping strategy, for example, preferring to avoid public places and private invitations. Instead the informants adopted a supportive role, often remaining at home with their wives. Chemically sensitive persons generally favour avoidance as an effective coping strategy $[10,13,16]$, which is also recommended by the majority of Danish General Practitioners (GP's) [21]. However, it is important to distinguish between the physical and the psychological aspects. At a first consultation, a GP's focus is mainly on the physical aspects. When patients complain of inconveniences, which in some cases are severe, caused by the inhalation of specific airborne chemicals, the GP's primary goal is to find a practical solution to their problems. Here, avoidance is the obvious choice, for example, avoiding fragranced products. Whereas if the social aspects were highlighted, avoidance should be recommended with caution as it could lead to social isolation with the ensuing risk of additional psychological problems. Nordin et al. [16] point out that the health services refrain from recommending avoidance, even though patients experience it as an effective method to reduce symptoms, as it could lead to further isolation. From a health perspective, the use of avoidant strategies in MCS is thus a complex issue, since it may have a serious impact on the individual life situation as well as on the family. As such, insight into 
the pathophysiological mechanisms of MCS and evidencebased treatments are highly needed.

Economy was another main theme in the focus groups. In the study by Söderholm and Nordin[14] the participants talked about economic limitations, where the spouses were responsible for generating the main income. Our findings are in agreement with this study, with our informants acknowledging lack of finances and economic burdens. It is increasingly being recognized that the impact on spouses and families who live with chronically ill persons goes beyond the consequences of caregiving [15]. Studies on spouses of chronically ill patients have revealed the need for involvement, not only in acquiring knowledge of the illness itself, but also in management guidance $[15,17,22,23]$.

In contrast to our findings, a study on female partners' experiences living with a fibromyalgic spouse suggested that they felt excluded from their husbands emotions [22]. Our informants expressed no such feelings, suggesting that women (female patients) are perhaps more inclined to express their emotions, thus involving their spouses in their situation rather than excluding them. Dogan et al. have described how Turkish female patients with fibromyalgia tended to continue their household and childcare activities despite their chronic illnesses, while their husbands continued in their traditional roles [23]. Culture may thus have a practical importance on the relationships. Household jobs and shopping were once women's domains, but our overall impression is that the male participants had accepted their situations and were not bound by such traditions. It was a "we" situation to succeed.

Some limitations must be considered when interpreting our findings. The findings are a reflection of the focus groups' opinions, and, as such, other spouses of chemically sensitive persons may cope differently. The informants were strangers to each other, which may have led to a more open and relaxed discussion, where participants were not later held responsible for their remarks. The collection of data and subsequent analyses were carried out within a short period, which can be beneficial for the results and focus, thus avoiding loss of interest in the theme or changing en route.

\section{Conclusion}

MCS may impose considerable strain on the family. The extra costs and efforts to avert problems were demanding and likely to contribute to social isolation. The use of different coping strategies appeared to be vital and possibly crucial to the relationship's survival. It is likely that spouses of chemically sensitive women would benefit from attending support groups. However, more studies on the impact of MCS on affected families are needed.

\section{Conflict of Interests}

The authors declare that there is no conflict of interests.

\section{References}

[1] R. A. Graveling, A. Pilkington, J. P. K. George, M. P. Butler, and S. N. Tannahill, "A review of multiple chemical sensitivity,"
Occupational and Environmental Medicine, vol. 56, no. 2, pp. 73-85, 1999.

[2] N. D. Berg, A. Linneberg, A. Dirksen, and J. Elberling, "Prevalence of self-reported symptoms and consequences related to inhalation of airborne chemicals in a Danish general population," International Archives of Occupational and Environmental Health, vol. 81, no. 7, pp. 881-887, 2008.

[3] C. Hausteiner, S. Bornschein, J. Hansen, T. Zilker, and H. Förstl, "Self-reported chemical sensitivity in Germany: a population-based survey," International Journal of Hygiene and Environmental Health, vol. 208, no. 4, pp. 271-278, 2005.

[4] A. Johansson, A. Bramerson, E. Millqvist, S. Nordin, and M. Bende, "Prevalence and risk factors for self-reported odour intolerance: the Skovde population-based study," International Archives of Occupational and Environmental Health, vol. 78, no. 7, pp. 559-564, 2005.

[5] R. Kreutzer, R. R. Neutra, and N. Lashuay, "Prevalence of people reporting sensitivities to chemicals in a populationbased survey," American Journal of Epidemiology, vol. 150, no. 1, pp. 1-17, 1999.

[6] C. Winder, "Mechanisms of multiple chemical sensitivity," Toxicology Letters, vol. 128, no. 1-3, pp. 85-97, 2002.

[7] S. M. Caress and A. C. Steinemann, "Prevalence of multiple chemical sensitivities: a population-based study in the southeastern United States," American Journal of Public Health, vol. 94, no. 5, pp. 746-747, 2004.

[8] S. M. Caress and A. C. Steinemann, "A national population study of the prevalence of multiple chemical sensitivity," Archives of Environmental Health, vol. 59, no. 6, pp. 300-305, 2004.

[9] F. Eek, B. Karlson, K. Österberg, and P. O. Östergren, "Factors associated with prospective development of environmental annoyance," Journal of Psychosomatic Research, vol. 69, no. 1, pp. 9-15, 2010.

[10] P. R. Gibson, A. N. M. Elms, and L. A. Ruding, "Perceived treatment efficacy for conventional and alternative therapies reported by persons with multiple chemical sensitivity," Environmental Health Perspectives, vol. 111, no. 12, pp. 14981504, 2003.

[11] P. R. Gibson, J. Cheavens, and M. L. Warren, "Social support in persons with self-reported sensitivity to chemicals," Research in Nursing and Health, vol. 21, no. 2, pp. 103-115, 1998.

[12] P. R. Gibson, L. G. Sledd, W. H. Mcenroe, and A. P. Vos, "Isolation and lack of access in multiple chemical sensitivity: a qualitative study," Nursing and Health Sciences, vol. 3, no. 3, pp. 232-237, 2011.

[13] S. Skovbjerg, S. Brorson, A. Rasmussen, J. D. Johansen, and J. Elberling, "Impact of self-reported multiple chemical sensitivity on everyday life: a qualitative study," Scandinavian Journal of Public Health, vol. 37, no. 6, pp. 621-626, 2009.

[14] A. Söderholm and S. Nordin, "The experience of living with sensory hyperreactivity-accessibility, financial security, and social relationships," Health Care for Women International, vol. 32, no. 8, pp. 686-707, 2011.

[15] A. N. Baanders and M. J. W. M. Heijmans, "The impact of chronic diseases: the partner's perspective," Family and Community Health, vol. 30, no. 4, pp. 305-317, 2007.

[16] M. Nordin, L. Andersson, and S. Nordin, "Coping strategies, social support and responsibility in chemical intolerance," Journal of Clinical Nursing, vol. 19, no. 15-16, pp. 2162-2173, 2010.

[17] S. Söderberg, M. Strand, M. Haapala, and B. Lundman, "Living with a woman with fibromyalgia from the perspective 
of the husband," Journal of Advanced Nursing, vol. 42, no. 2, pp. 143-150, 2003.

[18] H. Flor, D. C. Turk, and O. B. Scholz, "Impact of chronic pain on the spouse: marital, emotional and physical consequences," Journal of Psychosomatic Research, vol. 31, no. 1, pp. 63-71, 1987.

[19] S. Kvale, Interview-En Introduktion til det Kvalitative forsknings Interview, Hans Reitzels, 8th edition, 2002.

[20] B. Halkier, Fokusgrupper, Samfundslitteratur, 2nd edition, 2008.

[21] S. Skovbjerg, J. D. Johansen, A. Rasmussen, H. Thorsen, and J. Elberling, "General practitioners' experiences with provision of healthcare to patients with self-reported multiple chemical sensitivity," Scandinavian Journal of Primary Health Care, vol. 27, no. 3, pp. 148-152, 2009.

[22] M. Paulson, A. Norberg, and S. Söderberg, "Living in the shadow of fibromyalgic pain: the meaning of female partners' experiences," Journal of Clinical Nursing, vol. 12, no. 2, pp. 235-243, 2003.

[23] S. K. Dogan, Y. K. Aytur, and C. Atbasoglu, "Assessment of the relatives or spouses cohabiting with the fibromyalgia patients: is there a link regarding fibromyalgia symptoms, quality of life, general health and psychologic status?" Rheumatology International, vol. 1, no. 9, pp. 1137-1142, 2010. 


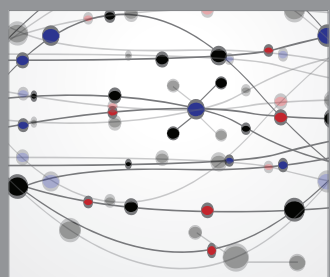

The Scientific World Journal
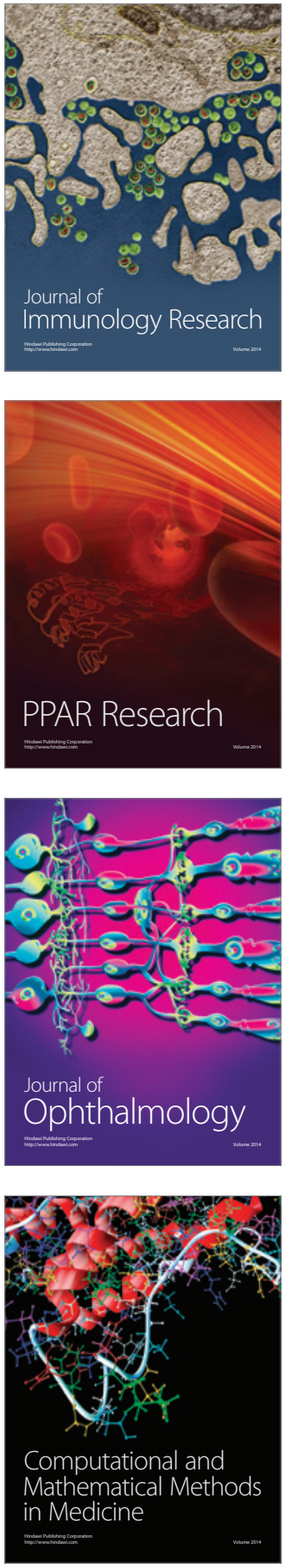

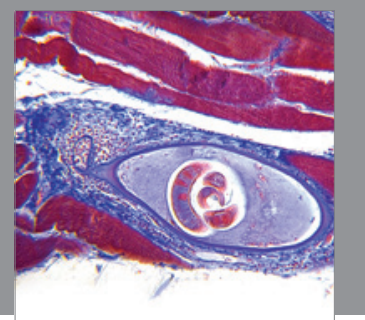

Gastroenterology

Research and Practice
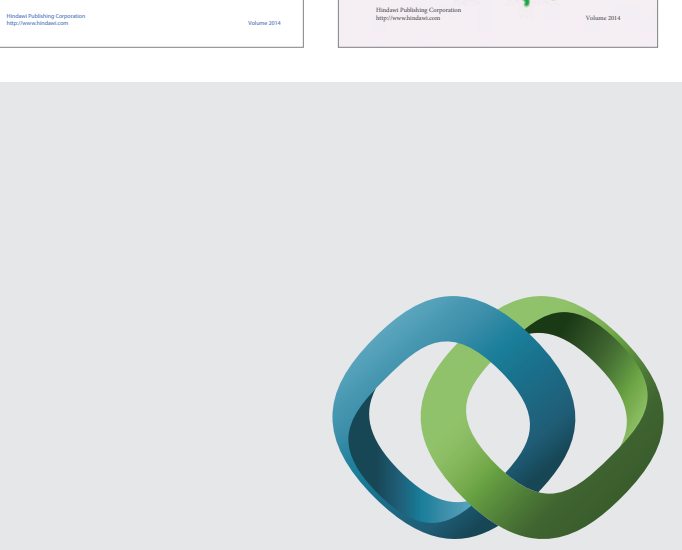

\section{Hindawi}

Submit your manuscripts at

http://www.hindawi.com
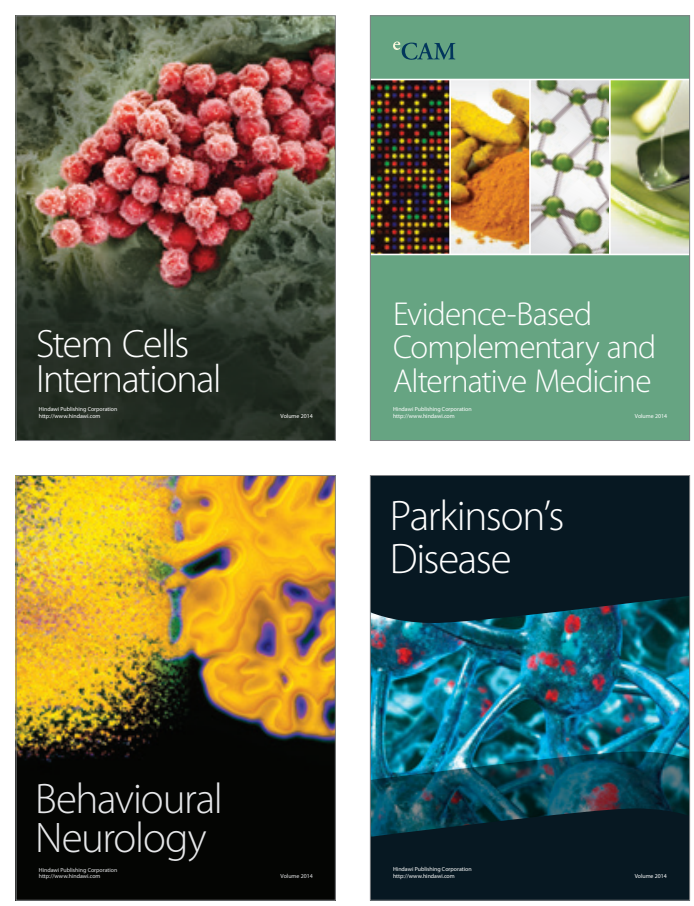

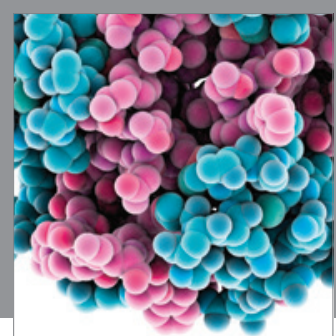

Journal of
Diabetes Research

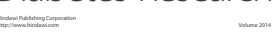

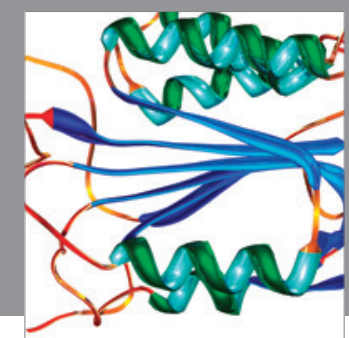

Disease Markers
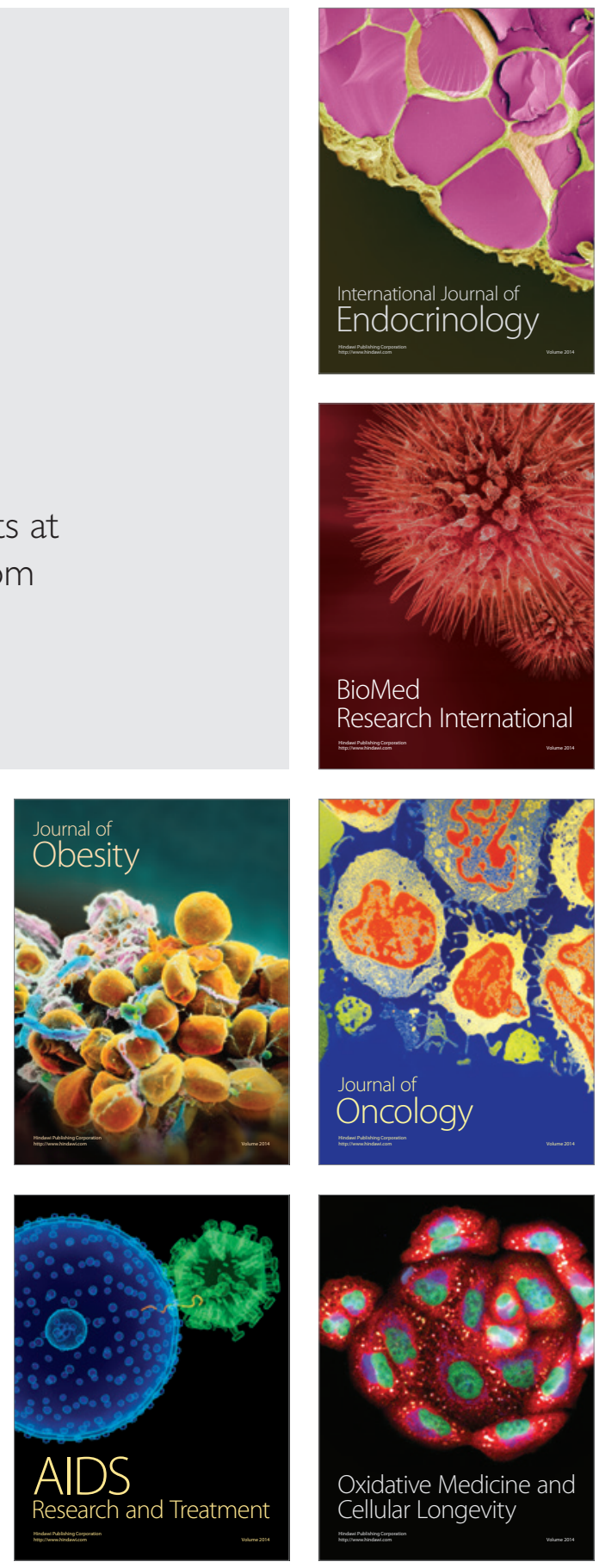